Hart, B.L., \& Leedy, M.G. (1983). Female sexual responses in male cats facilitated by olfactory bulbectomy and medial preoptic/anterior hypothalamic lesions. Behavioral Neuroscience, 97(4): 608-614. Published by the American Psychological Association (ISSN: 1939-0084).

\title{
Female Sexual Responses in Male Cats Facilitated by Olfactory Bulbectomy and Medial Preoptic/Anterior Hypothalamic Lesions
}

\author{
Benjamin L. Hart and Mitzi G. Leedy
}

\begin{abstract}
Bilateral lesions of the medial preoptic/anterior hypothalamic area and olfactory bulbectomies were performed sequentially in sexually active male cats, with the order of operations balanced. Male sexual behavior was virtually eliminated by the combined operations in all cats. Increased levels of female preceptive behavior were seen following either type of lesion alone, and combined operations resulted in increased levels of receptivity. The results support the concept that there exists, within the brains of male animals, the neural basis for the display of female as well as male sexual responses and that certain brain operations may potentiate the display of female responses. However, it does not appear that the reduction of male behavior is necessarily coupled with an increase in female behavior since no correlation was seen between the degree of enhancement of female behavior and the decrement in male behavior.
\end{abstract}


Sexual responses typical of the opposite sex are on occasion displayed by both male and female animals. This phenomenon is most extensively documented in rats. Estrogen treatment of male rats castrated in adulthood has been found to evoke the display of lordosis when the subjects are approached or mounted by stud males (Davidson \& Block, 1969; Larsson, Sodersten, Beyer, Morali, \& Perez-Palacios, 1976; Sodersten \& Larsson, 1975; van de Poll \& van Dis, 1977). Conversely, mounting, intromission, and even ejaculatory responses have been observed in female rats given long-term androgen or estrogen treatment (Emery \& Sachs, 1975; Krieger \& Barfield, 1976). Our ability to generalize the concept of a bisexuality in the representation of the neural basis of sexual behavior beyond the laboratory rat rests with exploration of this concept in other species.

Certain brain lesions may release the inhibition upon the neural circuitry underlying sexual responses of the opposite sex, so that with appropriate hormonal treatment the corresponding sexual behavior may be more readily displayed. One purpose of the present study was to explore the ease with which feminine preceptive (Beach, 1976), or solicitous, behavior and receptive responses could be evoked by estrogen treatment in male cats castrated in adulthood. The second purpose was to determine whether brain lesions might potentiate this effect. One such operation that would be likely to increase female responses is the removal of the olfactory bulbs. In the female rat, for example, olfactory bulbectomy potentiates the ability of estrogen treatment to evoke the display of lordosis (Al Satali \& Aron, 1977; Edwards \& Warner, 1972; Moss, 1971). Olfactory bulbectomy produces a slight, but significant, enhancement of lordosis intensity in male rats given estrogen and progesterone (Edwards \& Warner, 1972). It is possible that the olfactory bulbs have a tonic suppressive effect on the display of female sexual responses in both male and female animals and removal of the bulbs or severing of the olfactory 
tracts may have a disinhibitory effect.

Work on rodents also suggests that bilathypothalamic (MP-AH) area enhance the tendency of females to display lordosis when given estrogen treatment (Nance, Christenson, Shryne, \& Gorski, 1977; Powers

\& Valenstein, 1972). In both male and female guinea pigs, MP-AH lesions facilitate lordotic responding (Rodriguez-Sierra \& Terasawa, 1979). It is well known that MP-AH lesions eliminate or greatly reduce male copulatory behavior in male cats as in males of all other species studied (Hart, Haugen, \& Peterson, 1973).

The possibility that brain operations that markedly impair male sexual behavior can facilitate the display of female sexual responding brings up the issue of whether the neural substrates of the two systems of sexual behavior are coupled such that the degree of impairment in male sexual responding is correlated with the degree of enhancement of female sexual behavior. An approach to this question was attempted in the present study by testing male cats for both male sexual behavior and female sexual responding preoperatively and postoperatively so that impairment of male sexual behavior could be correlated within individual subjects.

In the present study, both olfactory bulbectomy and MP-AH lesions were performed sequentially in a counterbalanced design to examine the effect of these lesions in potentiating the display of female behavior by male cats given estrogen treatment.

\section{METHOD}

\section{Subjects}

Male cats of mixed breed, which were obtained as adults from the campus Animal Resource Services, were screened for copulatory behavior, and 23 animals that copulated in at least 6 of the 10 most recent tests with a receptive female were included in the experiment. Preoperative tests for male sexual behavior were 
conducted while the males were gonadally intact or following castration and receiving injections of $5 \mathrm{mg} /$ $\mathrm{kg}$ testosterone propionate (TP) every $48 \mathrm{hr}$ or an sc implantation with Silastic tubing, (ID, $1.57 \mathrm{~mm}$; OD, $3.18 \mathrm{~mm}$ ) into which was packed crystalline testosterone for a length of $50 \mathrm{~mm}$. Some of these males had previously been castrated as part of an earlier study which determined that these types of testosterone replacement will maintain serum testosterone concentrations that exceed those of gonadally intact male cats and maintain normal sexual behavior (Hart, Note 1).

The subjects were housed in colony pens of six to eight cats with individual perches provided for each cat. While receiving estrogen injections and testing for the display of female sexual responses, however, the males were housed in individual cages $m$ the same room. Food and water were available at all times.

\section{Surgical Procedures}

Anesthesia for both the olfactory bulbectomy and the performance of MP-AH brain lesions was induced by sodium thiamylal, and surgical anesthesia was maintained by the inhalant anesthetic Fluothane. Heart rate, respiratory rat ${ }^{\star}$, and body temperature were monitored with a modified Beckman Vital Signs Monitor and maintained within optimal limits by regulating the anesthetic level.

The surgical procedure for olfactory bulbectomy (OB) involved completely severing the olfactory tracts that run between the olfactory bulbs and the forebrain, plus removing approximately the caudal two thirds of the olfactory bulb (the rostral one third of the olfactory bulb is inaccessible.) The surgical procedure is the same as that used by Aronson and Cooper (1974) as described in more detail elsewhere (Hart, 1981). Briefly, an opening 6-8 $\mathrm{mm}$ in diameter was made in the roof of the frontal sinus, and after removal of the midsagittal septum, a hole was made in the floor of the frontal sinus to expose the olfactory fossa Most of the olfactory bulbs was then aspirated with a 14-ga. metal suction cannula, and the olfactory tracts were completely severed. For those subjects receiving sham olfactory bulbectomies, an opening was made into the frontal sinus, and the midsagittal septum was removed; however, the olfactory fossa was not exposed. 
The stereotaxic procedure for making bilateral MPAH lesions has been reported elsewhere (Hart el al.t 1973; Hart \& Ladewig, 1979). X-ray ventnculography, obtained by the injection of a radiopaque contrast medium (.1 ml of meglumme iothalamate, $60 \%$ ) into the third ventricle, was used to ensure accurate lesion placement. The electrode was directed so as to pass through the caudal edge of the anterior commissure and to rest $1 \mathrm{~mm}$ above the optic chiasm, 1.0-1.5 mm lateral to the midline. These electrode placements were confirmed by radiographs prior to making lesions. Radio-frequency lesions were made with a Kopf Model K 13882 electrode with a thermistor tip. The tip was .7 mm in diameter and had an exposed length of $1.5 \mathrm{~mm}$. The lesions were made with the Kopf Model RFG-4 radio-frequency generator. Current was raised gradually over $30 \mathrm{sec}$ until the tissue at the electrode tip was $68^{\circ} \mathrm{C}$, and the tissue was maintained at this temperature for $1 \mathrm{~mm}$. The procedure for sham MPAH operations was the same except that the electrode was lowered only to the level of the anterior commissure and no current was passed.

Following surgery for each operation, subjects were allowed to recover in individual cages for at least 1 wk. All subjects were usually alert and eating normally by the end of this postoperative period. On occasion, subjects showed little interest in food after the olfactory bulbectomy. These animals were induced to eat by being fed a highly palatable cat food-placing the food in the mouths of these animals if necessary.

\section{Behavioral Testing Procedures}

All tests were conducted in a semiacoustically insulated $2 \times 2 \mathrm{~m}$ test chamber with a one-way glass window for observation. The test chamber was located in the animal colony room. An exhaust fan in the chamber provided a sound masking noise. Stimulus females used for testing male sexual behavior were brought into behavioral estrus by the administration of two injections of $.25 \mathrm{mg}$ of estradiol cypionate (ECP) given $48 \mathrm{hr}$ apart.

Tests for male behavior. Subjects were placed in the test chamber a few minutes prior to the introduction of the female. The test began after the female was placed in the chamber. Observations were made on the occurrence of a properly oriented mount, pelvic 
thrusting, and intromission. A copulatory cry of the stimulus female helped to verify an intromission. All tests lasted $15 \mathrm{~mm}$ and were administered every 1-3 days.

For purposes of obtaining a graded score of male sexual behavior that could be used for correlation with changes in female sexual responding, a composite score was obtained for each subject by assigning one point each, per test, for mounting, pelvic thrusting, and intromitting. Thus for a series of 10 tests, the maximum composite score would be 30 .

Tests for female behavior. These tests were conducted after the animals had been castrated or had received no exogenous testosterone for at least 30 days. Each subject was given three injections of $.5 \mathrm{mg}$ of ECP at 2-day intervals Subjects had been isolated for 2 wk prior to receiving estrogen injections. Tests for female sexual response began 2 days after the third ECP injection.

The stud males used for testing the experimental subjects were allowed to mate with sexually receptive females from time to time to maintain their interest in mounting the test males. Preliminary observation had shown that extensive use of stud males with experimental males would diminish their sexual vigor. Therefore, in each testing period only three tests for female behavior were given to each of the experimental males. At least two stud males were used during a series of tests. If an encounter between the stud male and the experimental male was totally agonistic, the test was terminated immediately. If the subject showed either no receptivity or only very slight receptivity, the test was terminated after $5 \mathrm{~min}$. Otherwise, all tests lasted $15 \mathrm{~min}$. Measures were taken relating to both proceptive and receptive behavior. The following preceptive measures were taken: (a) approach to the stud if not done in an aggressive manner and (b) rubbing shoulders against the stud male in a solicitous fashion (neck-grip solicitation) or rubbing and rolling on the floor of the test chamber. These proceptive behaviors are typically displayed by female cats in behavioral estrus, and they often elicit mounts from the males. Measures of receptive responses to a stud male mount attempt were as follows: (a) crouching of the front legs and shoulders while elevating the pelvic region (pelvic elevation), (b) alternate stepping movements of the hind legs (treading), and (c) movement of the tail to one side or another (tail deviation). 
A composite score for proceptive behavior displayed during each of the series of tests was obtained by giving one point, per test, for approach to the stud male and one point for rolling and rubbing or neckgrip solicitation. The maximum obtainable score for proceptivity therefore was six. A composite score for receptivity was obtained by giving one point each, per test, for pelvic elevation, treading, and tail deviation.

The maximum possible score for receptivity was nine. Response to manual stimulation. Immediately prior to each test for female sexual behavior, all male animals were tested for their response to manual stimulation of the anogenital region. While the scruff of the neck was held, pressure was applied to the lower back of the animal, and the perigenital region- was stroked. The occurrence of pelvic elevation, tail deviation, and treading was recorded. The same scoring procedure was used as noted for receptivity.

\section{Schedule of Testing and Operative Procedures}

Males were initially given 10 tests for male sexual behavior at 1-3 day intervals. At least two females were used during this series. Following the conclusion of the last test, the subjects were castrated or testosterone therapy was terminated, and the animals rested for $4 \mathrm{wk}$. All subjects were isolated for $2 \mathrm{wk}$ before receiving injections of estrogen and testing for female sexual responding with a stud male. Following the third test for female sexual responses, and commencing at least 2 wk after the third ECP injection, subjects were divided into three groups, and surgery for MPAH lesions $(n=9)$, olfactory bulbectomy $(n=7)$, or sham lesions $(n=7)$ was performed. Between 14 and 21 days after surgery, all subjects were again tested for female sexual responding as before.

The operation for MP-AH lesions or olfactory bulbectomies was then performed on subjects of opposite groups approximately $2 \mathrm{wk}$ after the third ECP injection. Those subjects receiving sham olfactory bulbectomies then received sham lesions, and vice versa. After 14-21 days of recovery from the second operation, all subjects again received three injections of ECP and were given a third series of tests for female behavior as before. Two weeks after' the third ECP injection, subjects were given a testosterone implant or started on TP injections and were returned to the colony pen for postoperative testing for male sexual 
behavior 3-4 wk later. Ten tests, as before, with receptive females were conducted. This testing schedule is depicted in Figure 1.

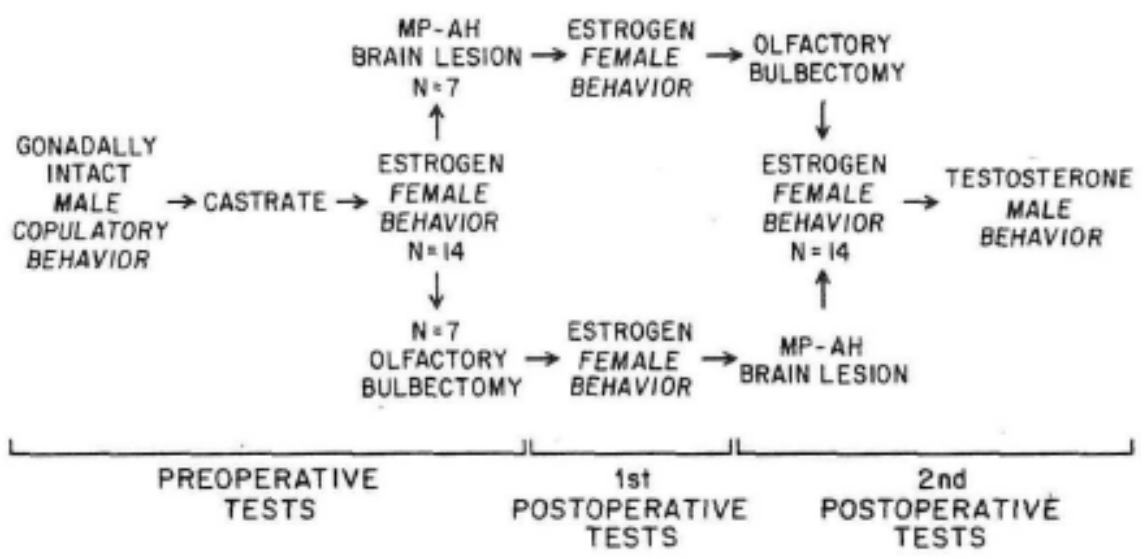

Figure 1. Schedule of operations and behavior testing. (Animals receiving sham operations followed the same sequence, with four receiving the sham olfactory bulbectomy first and three receiving the sham medial preoptic/anterior hypothalamic lesion first.)

\section{Postmortem Examination of the Brain}

All subjects were anesthetized, and the brains were perfused with physiological saline followed by neutralized formalin. The olfactory fossa and frontal poles of the brain were examined to verify the completeness of the olfactory tractotomy and the olfactory bulbectomy. The brains were removed and stored in formalin and later sectioned at $50 \mathrm{jim}$ on a freezing microtome. Representative sections, at about $.2 \mathrm{~mm}$ throughout the lesion area, were stained with cresyl violet and examined for location and extent of the lesions. The data from two animals that received the MP-AH lesions first were discarded from the experiment due to incorrect lesion placement; this left seven subjects in each of the three groups.

\section{RESULTS}

Data from testing of both male sexual behavior and female sexual responses are from only those animals in which the MPAH lesions were found to be in the designated area (and as confirmed by the marked impairment or loss of male copulatory behavior) 
and in which examination of the confirmed that the olfactory tract was completely severed. Figure 2 shows a typical lesion at its maximum extent.

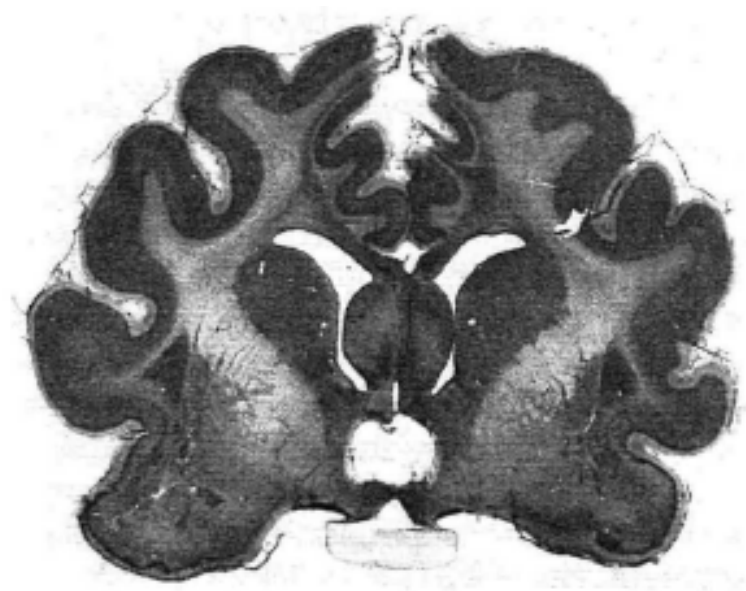

Figure 2. Photomicrograph of a medial preoptic/anterior hypothalamic area lesion in a male cat that showed an elimination of male sexual behavior and an increase in female sexual behavior.

Male sexual behavior was evaluated preoperatively and after each subject had sustained both olfactory bulbectomy and MPAH lesion or both sham operations. As expected, there was a very marked reduction in male copulatory behavior in those animals receiving the combined experimental surgeries. Sham-operated animals continued to show high levels of male behavior.

Of 14 subjects with lesions and olfactory bulbectomies, 9 continued to mount on occasion in postoperative testing, but only 1 of these subjects achieved an intromission on any of the tests, and this was only in one test. This reduction in copulatory performance was evident in a mean reduction in the composite male sexual behavior score from 25.0 to 3.4. An analysis of variance revealed that this change was highly significant, $\mathrm{F}(\mathrm{l}, 12)=262.6, p<.001$.

There was a general increase in the display of female sexual behavior following the experimental surgeries. Photographs of 
femalelike behavior by male cats that had received both MP-AH lesions and olfactory bulbectomies are shown in Figures 3 and 4.

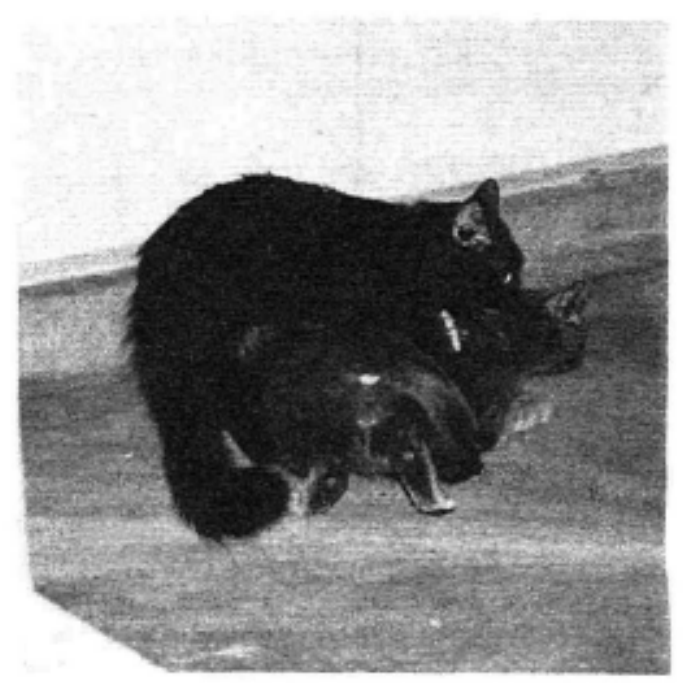

Figure 3. Photograph of an experimental male (gray) displaying pelvic elevation and tail deviation (receptive behavior) in response to the stud male's (black) neck grip and partial mount.

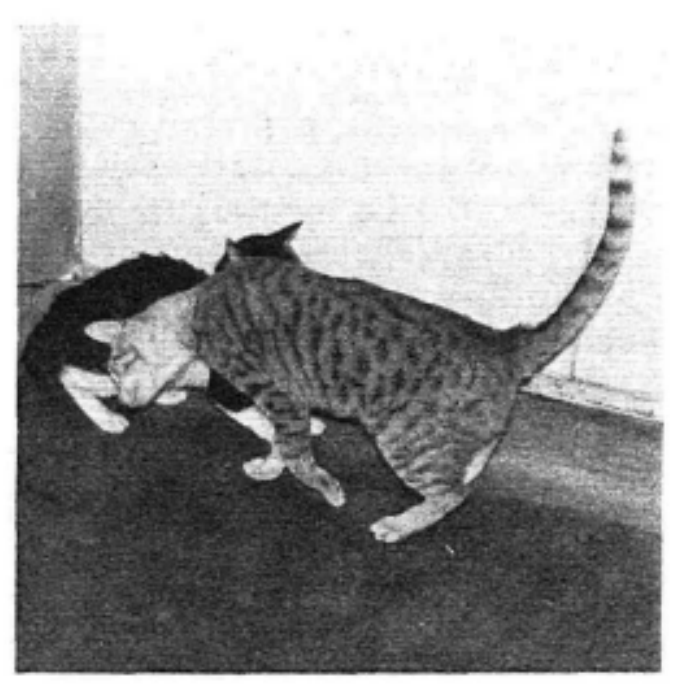

Figure 4. Photograph of an experimental male (foreground) rubbing against the stud male in a solicitous manner (proceptive behavior). 
The composite scores for both proceptivity and receptivity are presented in Figure

5. There was an enhancement in proceptivity after both the olfactory bulbectomy and the MP-AH operations in the first postoperative series of female behavior tests. An analysis of variance found no difference between the two experimental groups in proceptivity levels during the three series of tests, $\mathrm{F}(\mathrm{l}, 12)=4.54, p>.05$. Proceptivity did increase over test series for these two groups, $\mathrm{F}(2,24)=11.68, p<.01$. Subsequent Scheffe's tests showed that an increase was seen after the first operation regardless of type, $F(2,24)=5.24, p<.05$.

No further significant enhancement of proceptivity was observed following the second operation. Proceptivity also increased in those animals receiving sham operations; however, this increase was seen only when comparing the second postoperative tests with the preoperative tests, $F(2,12)=7.72$, $p<.01$. 

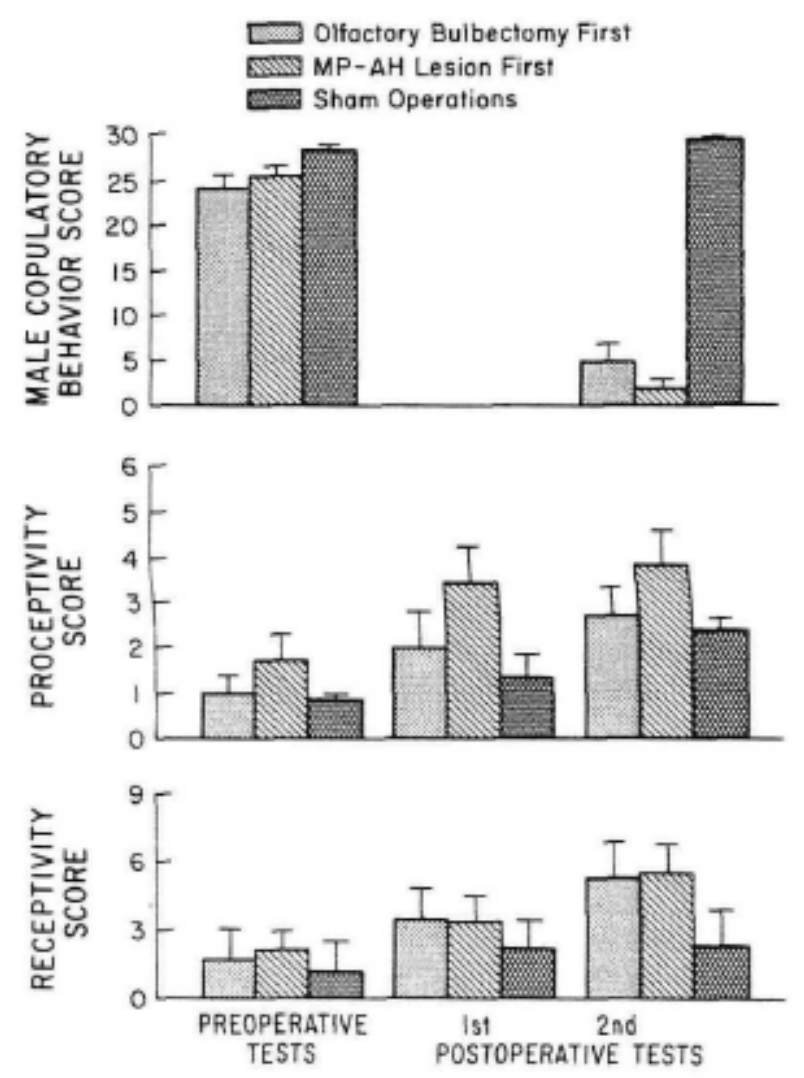

Figure 5. Graph of mean $( \pm S E$ ) preoperative and postoperative male copulatory behavior scores and mean female proceptive and receptive behavior scores for each group. (MP-AH = medial preoptic/anterior hypothalamic.)

Receptivity levels also increased during the postoperative testing series. Again, an analysis of variance found no difference between the two experimental groups, but there was a significant effect due to testing series, $F(2,24)=8.19, p<.01$. Subsequent Scheffe's tests showed that only after the second experimental operation was the increase in receptivity significant, $F(2,24)=$

$8.11, p<.01$. Those animals receiving two control operations did not show an increase in receptivity, $F(2,12)=2.46, p>.05$.

The composite scores for manual stimulation did not change across tests, $F(l, 12)$ $=.25, \mathrm{p}>.1$, nor did the groups differ among themselves, $F(2,24)=.81, p>.1$. 
The mean composite scores for the three test series were 8.7, 8.0, and 7.6 for those animals receiving the olfactory bulbectomy first; $8.3,8.4$, and 8.6 for those receiving the lesion first; and 4.9, 6.9, and 5.9 for those receiving the sham operations.

As can be seen from Figure 5, an increase in the mean composite scores of proceptivity and receptivity was accompanied by a marked decrease in the score for male copulatory behavior in those animals receiving olfactory bulbectomies and MP-AH lesions. In order to determine whether there was a correlation between the degree of decrease in the male behavior score and the increase in either proceptivity or receptivity among the individual subjects, a Spearman rank correlation coefficient was determined correlating both of these measures of female sexual responding with the changes in male behavior. The correlation coefficients for the change in proceptivity and receptivity with the change in male behavior were .1 and .2, respectively, and neither of these correlations approached the significance level.

\section{DISCUSSION}

The results of this study support the concept that there exists within the brains of male cats the neural basis for the display of both male sexual responses and female sexual responses. As evident in preoperative testing, the administration of estrogen to some male cats, castrated as adults, evokes at least, some elements of female sexual behavior. However, the levels of female sexual responding in those male cats did not approach the levels seen in female cats when treated with estrogen (Hart, 1971; Peretz, 1968; Whalen \& Hardy, 1970). Although proceptivity increased in all groups of animals in the present study, those receiving either MP-AH area lesions or olfactory bulbectomies showed an enhancement during the first postoperative test series, whereas those receiving sham 
operations did not show this effect until the second postoperative series of tests. Thus, it seems that repeated exposure to estrogen and the testing situation can result in an increased probability of a male cat's displaying proceptive behavior, but this effect can be facilitated by either the olfactory bulbectomy or the MP-AH lesion. Measures of receptivity displayed toward male stud cats were enhanced by the combined effect of both operations, but not by sham procedures. These findings are consistent with earlier observations on male rats and guinea pigs in which MP-AH lesions were found to potentiate the display of lordosis when injections of estrogen are given (Powers \& Valenstein, 1972; Rodriguez-Sierra \& Terasawa, 1979). Olfactory bulbectomy has been shown to facilitate the display of lordotic behavior by female rats by presumably removing the tonic inhibition (Al Satali \& Aron, 1977; Edwards \& Warner 1972; Moss, 1971). The olfactory bulbs may likewise be inhibitory to the display of female sexual behavior in males.

Response to manual stimulation of the anogenital region has been used as a measure of receptivity in cats (Villablanca, Marcus, \& Olmstead, 1976). The present study, while finding an increase in measures of receptivity and proceptivity, found no corresponding increase in responsiveness to such manual stimulation. Thus, we conclude that using an animal's response to manual stimulation is not a reliable measure of female sexual responses.

Although not tested in this study, it is known that olfactory bulbectomy in male cats does not impair male sexual behavior (Aronson \& Cooper, 1974; Hart, Note 2). Yet, in this study, olfactory bulbectomy was found to enhance measures of proceptivity and to potentiate the effects of MP-AH lesions on receptivity. Furthermore, since there was no correlation between the degree of impairment of male copulatory behavior and the degree of increase in either receptivity or proceptivity after the subjects had received both operations, it appears that 
the neural systems for male and female behavior are not coupled. Thus, the elements that sustain appropriate male sexual responding are not identical to those that suppress female responding.

\section{REFERENCE NOTES}

1. Hart, B. L. Unpublished observations, 1980.

2. Hart, B. L. Unpublished observations, 1981.

\section{REFERENCES}

Al Sataii, M., \& Aron, C. Influence of olfactory bulb removal on sexual receptivity in the rat. Psychoneuroendocnnology, 1977, 2, 399-407.

Aronson, L. R., \& Cooper, M. L. Olfactory deprivation and mating behavior in sexually experienced male cats. Behavioral Biology, 1974,11, 459-480.

Beach, F. A. Sexual attractivity, proceptivity, and receptivity in female mammals. Hormones and Behavior, $1976,7,105-138$.

Davidson, J. M., \& Bloch, G. J. Neuroendocrine aspects of male reproduction. Biology of Reproduction, 1969,1, 67-92.

Edwards, D. A., \& Warner, P. Olfactory bulb removal facilitates hormonal induction of sexual receptivity in the female rat. Hormones and Behavior, 1972, 3, 321-332.

Emery, D. E., \& Sachs, B. D. Ejaculatory pattern in female rats without androgen treatment. Science, 1975,190, 484-486.

Hart, B. L. Facilitation by estrogens of sexual reflexes in spinal cats. Physiology and Behavior, 1971, 7, 675-678

Hart, B. L. Olfactory tractotomy to control objectionable urine spraying and urine marking in cats. Journal of American Veterinary Medical Association, 1981,/ 79, 231-234. 
Hart, B. L., Haugen, C, \& Peterson, D. M. Effects of medial preoptic-anterior hypothalamic lesions on mating behavior of male cats. Brain Research, 1973, 54, 177-191.

Hart, B. L., \& Ladewig, J. Effects of medial preopticanterior hypothalamic lesions on development of sociosexual behavior in dogs. Journal of Comparative and Physiological Psychology, 1979,93,566-573.

Krieger, M. S., \& Barfield, R. J. Masculine sexual behavior- Pacing and ejaculatory patterns in female rats induced by electrical shock. Physiology and Behavior, 1976, 16, 671-675.

Larsson, K., Sodersten, P. Beyer, C. Morali, G., \& Perez-Palacios, G. Effects of estrone, estradiol and estriol combined with dihydrotestosterone on mounting and lordosis behavior in castrated male rate. Hormones and Behavior, 1976, 7, 379-390.

Moss, R. L. Modification of copulatory behavior in the female rat following olfactory bulb removal. Journal of Comparative and Physiological Psychology, 1971, 74, 374-382.

Nance, D. M., Christensen, L. W., Shryne, J. E., \& Gorski, R. A. Modifications in gonadotropin control and reproductive behavior in the female rat by hypothalamic and preoptic lesions. Brain Research Bulletin, 1977, 2, 307-312.

Peretz, E. Estrogen dose and the duration of the mating period in cats. Physiology and Behavior, 1968, 3, 41-43.

Powers, B., \& Valenstein, E. S. Sexual receptivity: Facilitation by medial preoptic lesions in female rate. Science, 1972,175,1003-1005.

Rodriguez-Sierra, J. F., \& Terasawa, E. Lesions of the preoptic area facilitate lordosis behavior in male and female guinea pigs. Brain Research Bulletin, $1979,4,513-517$.

Sodersten, P., \& Larsson, K. Lordosis behavior and mounting behavior in male rate: Effects of castration and treatment with estradiol benzoate or testosterone propionate. Physiology and Behavior, 1975,14, 159-164. 
van de Poll, N. E., \& van Dis, H. Hormone induced lordosis and its relation to masculine sexual activity in male rate. Hormone and Behavior, 1977, 8,1-7.

Villablanca, J. R., Marcus, R. J., \& Olmstead, C. E. Effects of caudate nuclei or frontal cortical ablations in cats: 1 . Neurology and gross behavior. Experimental Neurology, 1976, 52, 389-420.

Whalen, R. E., \& Hardy, D. F. Induction of receptivity in female rate and cats with estrogen and testosterone. Physiology and Behavior, 1970, 5, 529-533. 